\title{
Development of a cost-effective plastic scintillator for cosmic-ray muon radiography of a volcano
}

\author{
Yasushi Yamashina ${ }^{1}$, Tsuneo Yamashina ${ }^{1}$, Hideaki Taira ${ }^{2}$, and Hiroyuki K. M. Tanaka ${ }^{2,3}$ \\ ${ }^{1}$ CI Industry, 2-674-2, Mikkajima, Tokorozawa, Saitama 359-1164, Japan \\ ${ }^{2}$ Earthquake Research Institute, the University of Tokyo, 1-1-1 Yayoi, Bunkyo, Tokyo 113-0032, Japan \\ ${ }^{3}$ Atomic Physics Laboratory, RIKEN, 2-1 Hirosawa, Wako, Saitama 351-0198, Japan
}

(Received November 28, 2008; Revised March 25, 2009; Accepted March 30, 2009; Online published February 22, 2010)

\begin{abstract}
Muon radiography has recently made it possible to perform imaging of the internal structure of a volcano. Muon-detecting experiments were carried out at Asama and Usu volcanoes using a newly developed scintillator. The goal was to build a large detector that would improve the quality of the data collected by the muon radiography experimental set-up that is currently being used to study volcanic activities. To this end, a costeffective plastic scintillator was developed by a simple "heating-melting method". The detector consists of planes of scintillator strips and is currently in the prototyping phase. Using a cosmic-ray muon source, we found that the signal from our scintillator was consistent with the zenith-angle dependence of the cosmic-ray muon spectrum with no significant attenuation effects in the scintillator. We confirmed that our scintillators can be incorporated into a portable assembly-type cosmic-ray muon telescope system.
\end{abstract}

Key words: Cosmic-ray muon radiography, volcano, plastic scintillator, detection system.

\section{Introduction}

Cosmic-ray muon radiography is similar to X-ray radiography, with the exception that penetrating muons are used instead of X-rays. In terms of determining the internal structure of a volcano, this method measures the absorption of muons along a cross section of the volcano parallel to the plane of the detector, on which the average density along all muon paths is projected. The absorption of the muons by the internal structures of the volcano can then be used to determine the density profile of that volcano. Such measurements would be ideal for studying the time-dependent changes in the crustal structure at sites which cannot be well resolved because of their strong structural heterogeneity and potential difficulty to be accessed and which, therefore, are not amenable to having their structure determined by conventional electromagnetic or seismic techniques. Volcanoes make particularly good study targets for muon radiography because they are axi-symmetric, and it is reasonable to assume that the observed density variations are localized in the vent or crater area. The use of emulsion films (Tanaka et al., 2007a, b, c, 2008; Tanaka and Yokoyama, 2008) has recently made it possible to image the internal structure of a volcano. An emulsion film is a completely power-free particle tracking device that is light enough to be carried up a mountain. However, the image can be obtained only after the emulsion has been developed - at a later timesomewhat like a photographic film. In cosmic-ray muon radiography, a detector with an area on the order of square

Copyright (C) The Society of Geomagnetism and Earth, Planetary and Space Sciences (SGEPSS); The Seismological Society of Japan; The Volcanological Society of Japan; The Geodetic Society of Japan; The Japanese Society for Planetary Sciences; TERRAPUB.

doi:10.5047/eps.2009.03.003 meters is placed so that it is imaging a volume that is higher in elevation adjacent to the detector. The intensity of the image pixel in the detector is determined by the attenuation of the incident muons caused by absorption in the Earth's crust. This technique is utterly independent of the geophysical model and directly measures the density length (density $\times$ path length). By determining the path lengths from topographic information, the measurement gives provides researchers with the average density $\langle\rho\rangle$ along the path lines of cosmic-ray muons through the Earth.

The imaging techniques are aimed at detecting the cosmic-ray muons and are intricately associated with electronic systems and computers. The function of the scintillator in the imaging systems is to convert the energy deposited along the path of the high-energy muons into visible light. The experimental arrangement for a real-time volcano monitoring system requires a relatively large detection area to collect a large number of muon events. With these increasing requirements for volcanic imaging equipment, the demands made on the scintillator as the detection material in imaging systems will be enormous. About $320 \mathrm{~kg}$ of scintillator is required for a cosmic-ray muon detection system with a size of $2 \times 2 \mathrm{~m}$. In principle, an ideal scintillator for muon radiography should have the following properties: (1) high luminous efficiency; (2) short decay time; (3) no after glow; (4) short attenuation length; (5) low cost. However, in general, the choice of a scintillator for any given project is based on a compromise between light output and cost. Cast plastic scintillators may cost between $\$ 40-60$ per kilogram. Therefore, the development of new scintillators for cosmic-ray muon radiography will provide a major impulse to research on the diagnosis and monitoring of volcanoes. We report here our development of a new scintillator that is 
Table 1. Physical property of styrene.

\begin{tabular}{ll}
\hline Density $\left(\mathrm{g} / \mathrm{cm}^{3}\right)$ & $1.04-1.06$ \\
Refractive index & $1.58-1.62$ \\
Rate of transmission $(\% / 12 \mathrm{~cm})$ & 89 at $\lambda=420 \mathrm{~nm} ; 89.7$ at $\lambda=500 \mathrm{~nm}$ \\
\hline
\end{tabular}
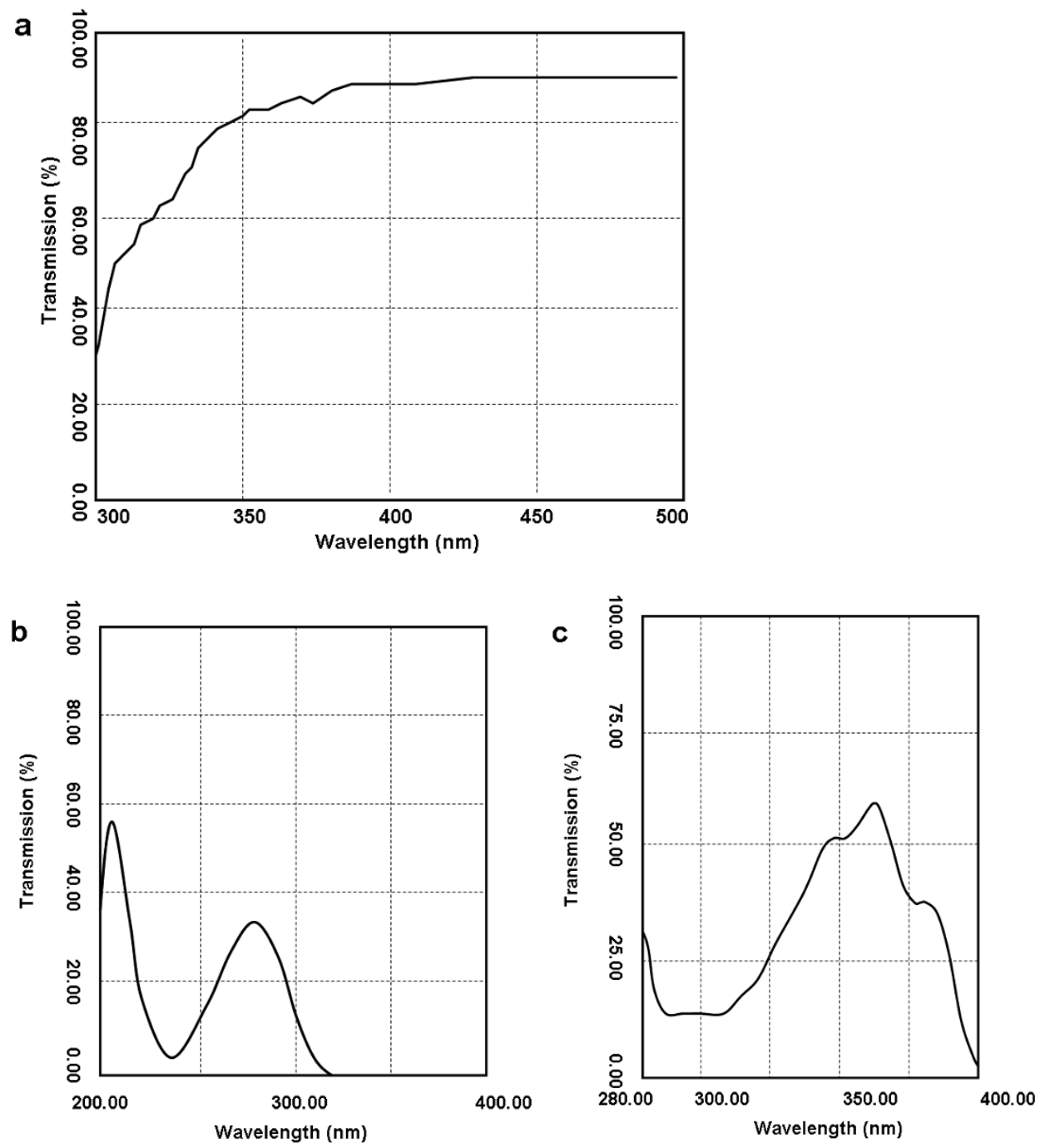

Fig. 1. Transmission rate of scintillator materials used in the scintillator sample (length $12 \mathrm{~cm}$ ): (a) polystyrene, (b) para-terphenyl, (c) POPOP.

based on a simple "heating-melting method"; this scintillator has a significantly lower price tag than those currently available.

\section{Scintillator Requirements for Cosmic-ray Muon Radiography}

In the field of cosmic-ray muon radiography, the energy of the muons detected is above few GeV. Any improvement of signal-to-noise ratio cannot be obtained by increasing the cosmic-ray source intensity but, rather, is achieved by increasing the sensitivity of detector. Tanaka et al. (2010) recently developed a portable assembly type cosmic-ray muon telescope module. The module comprises a plastic scintillator, acryl light guide, and power-effective photomultiplier tube (PMT) (Hamamatsu H 7724) in a polycarbonate container. At the observation site, 48 modules are arranged so as to construct two segmented scintillation de- tector planes by which to track muon trails. The length of the module has to be longer than $1 \mathrm{~m}$ in order to have a detector plane that is larger than $1 \mathrm{~m}^{2}$. However, the conventional cheap scintillators have a very short light attenuation length-usually shorter than $50 \mathrm{~cm}$. In order to improve the quality of the imaging, it is necessary to reduce the light attenuation effects in the detector.

\section{New Scintillator for Cosmic-ray Muon Radio- graphy}

Wavelength shifters (POPOP and P-Terphenyl) were prepared by melting a mixture of styrene monomers at 160$180^{\circ} \mathrm{C}$ for $18 \mathrm{~h}$ until they were completely hydrolyzed. The properties of styrene are summarized in Table 1 and Fig. 1(a). The melted mass was then allowed to cool in air and subsequently annealed in an electric furnace at $150^{\circ} \mathrm{C}$ for $15 \mathrm{~h}$. The amounts of styrene, para-Terphenyl, and 
Table 2. Feature of the present plastic scintillator.

\begin{tabular}{ll}
\hline Composition and properties of the plastic scintillator \\
(1) Base resin (PS) polystyrene $\left[\mathrm{C}_{8} \mathrm{H}_{8}\right]$ & $99 \%$ \\
(2) Para-terphenyl $\left(1,4\right.$ diphenyl benzene) $\left[\mathrm{C}_{18} \mathrm{H}_{14}\right]$ & $1 \%$ \\
(3) POPOP $\left(1,4-\mathrm{Bis}[2-5-\right.$ phenyloxazolyl] $)$-benzene $\left[\mathrm{C}_{24} \mathrm{H}_{16} \mathrm{~N}_{2} \mathrm{O}_{2}\right]$ & $0.03 \%$ \\
B. Specific density $\left(\mathrm{g} / \mathrm{cm}^{3}\right)$ & 1.04 \\
C. Wavelength of maximum scintillation & $420 \mathrm{~nm}$ \\
D. Decay constant & $3.0-3.2 \mathrm{~ns}$ \\
E. Scintillation efficiency $(\%$ Anthracene) & $50-60 \%$ \\
F. Light attenuation length & $110-90 \mathrm{~cm}$ \\
G. Refractive index & 1.5 \\
H. Softing point & $80-82^{\circ} \mathrm{C}$ \\
\hline
\end{tabular}
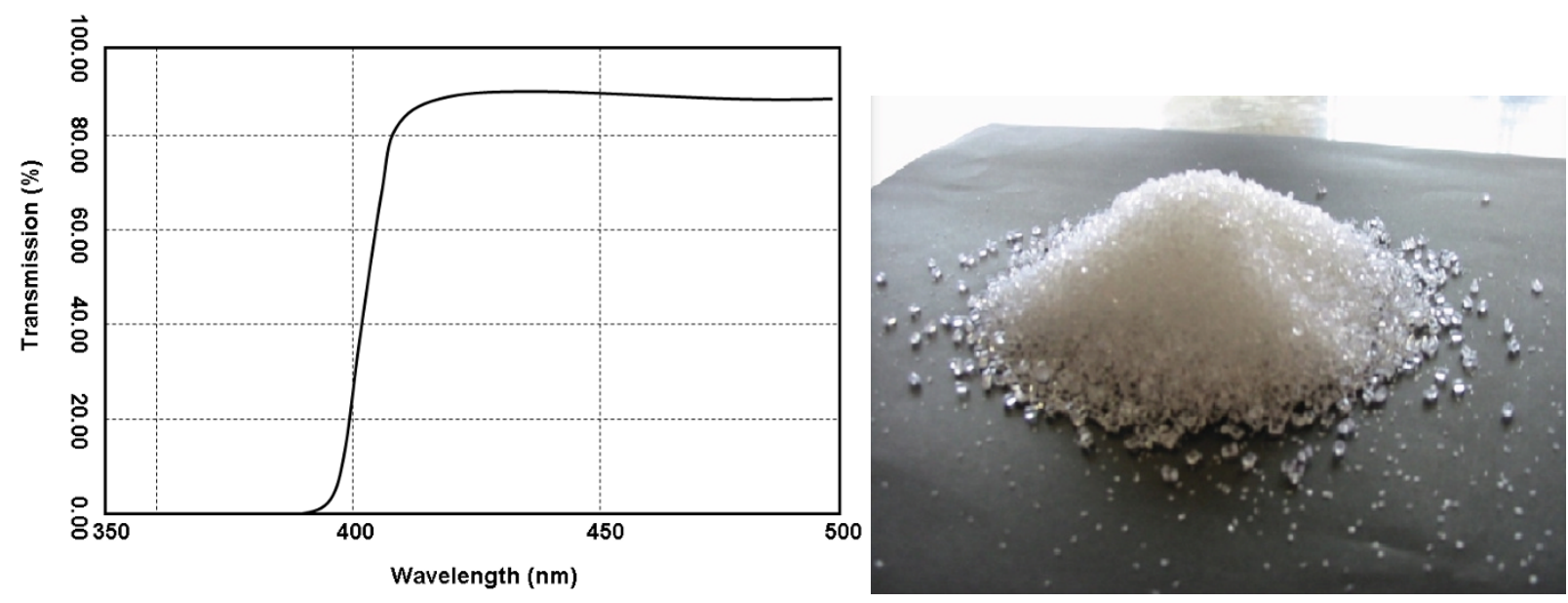

Fig. 2. Left: Transmission rate of the present plastic scintillator sample (length $12 \mathrm{~cm})$. Right: The produced pellet polymer.

POPOP in the samples are given in Table 2. The density of typical polystyrene ranges from 1.04 to 1.06 , and the reflection index ranges from 1.58 to 1.62 . The photon transmission rate for a scintillator sample with a length of $12 \mathrm{~cm}$ is $89 \%$ at $420 \mathrm{~nm}$ and $89.7 \%$ at $500 \mathrm{~nm}$. Plastic scintillator pellets with a diameter of $3 \mathrm{~mm}$ were formed by quenching the melts in a carbon steel mold. Once the pellets are produced, a scintillator can be constructed easily by heating and melting these pellets. Using this methodology, we constructed a plate scintillator measuring $50 \times 50 \times 5 \mathrm{~cm}$. The light yields and decay times of the new scintillator were tested with a ${ }^{60} \mathrm{Co} \gamma$-ray source. Densities were measured by the Archimedes method using deionized water as the immersing medium. The UV/VIS transmittance of the glass samples was measured with a UV-2550 spectrophotometer. The fluorescence spectra were also measured.

The densities are given in Table 2. The density of doped styrene is, essentially, the same as that of undoped styrene because the low amount of dopant does not change in density. Figure 2 shows the photon transmission spectra of our scintillator in which p-Terphenyl and POPOP are doped. The scintillator is opaque for ultraviolet: the photon transmission rate as for the scintillator sample with a length of $12 \mathrm{~cm}$ is $2 \%$ for a wavelength of $400 \mathrm{~nm}$ and $77 \%$ for one of $420 \mathrm{~nm}$. Compared with the photon transmission spectra of styrene without doping (Fig. 1(a)), we find that photon transmission is not affected by such doping. For reference, Fig. 1(b) shows the photon transmission spectra for
p-Terphenyl and Fig. 1(c) that for POPOP, with peaks at $277.2 \mathrm{~nm}$ and $362.4 \mathrm{~nm}$, respectively. The signal shape was evaluated from an oscilloscope picture. The time width of the pulses is consistent with a nanosecond-type fast response of the fluors. The decay time of the doped glasses was estimated to be 3.0-3.2 ns. Compared with the decay time of scintillating glass, that of our scintillator is little longer, but it is short enough for making a coincidence signal from cosmic-ray muons. Mechanical strength was also measured. The measured bending strength, ultimate strength for tension, and compression were 60, 55, and $800 \mathrm{MPa}$. Other features of our new scintillator are summarized in Table 2.

\section{Assembly Testing}

A series of tests led to the development of a new scintillator for cosmic-ray muon radiography to ensure the efficient transmission of light through the optical connectors. We tested this scintillator to confirm if it was applicable for use with the segmented muon telescope for muon radiography of a volcano. The muon telescope consists of 7-cm-wide crossed segmented scintillator strips $\mathrm{cm}$ aligned in the direction of a volcano; these strips allow for tracking of the muons after the muons pass through the mountain. A straight line connecting the intersecting points of muons at two detector planes determines the muon trail (Tanaka et al., 2010). To test the scintillator, we attached a 2 -inch photomultiplier tube (PMT: Hamamatsu R7724) to the end 
$\mathbf{a}$

Detector 1

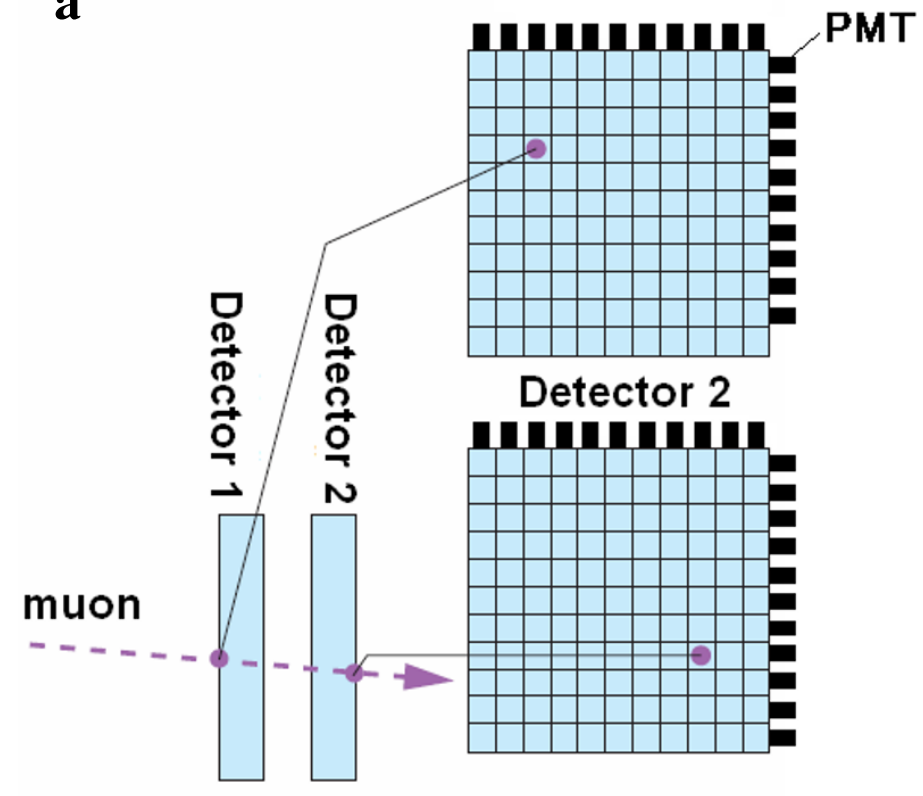

b

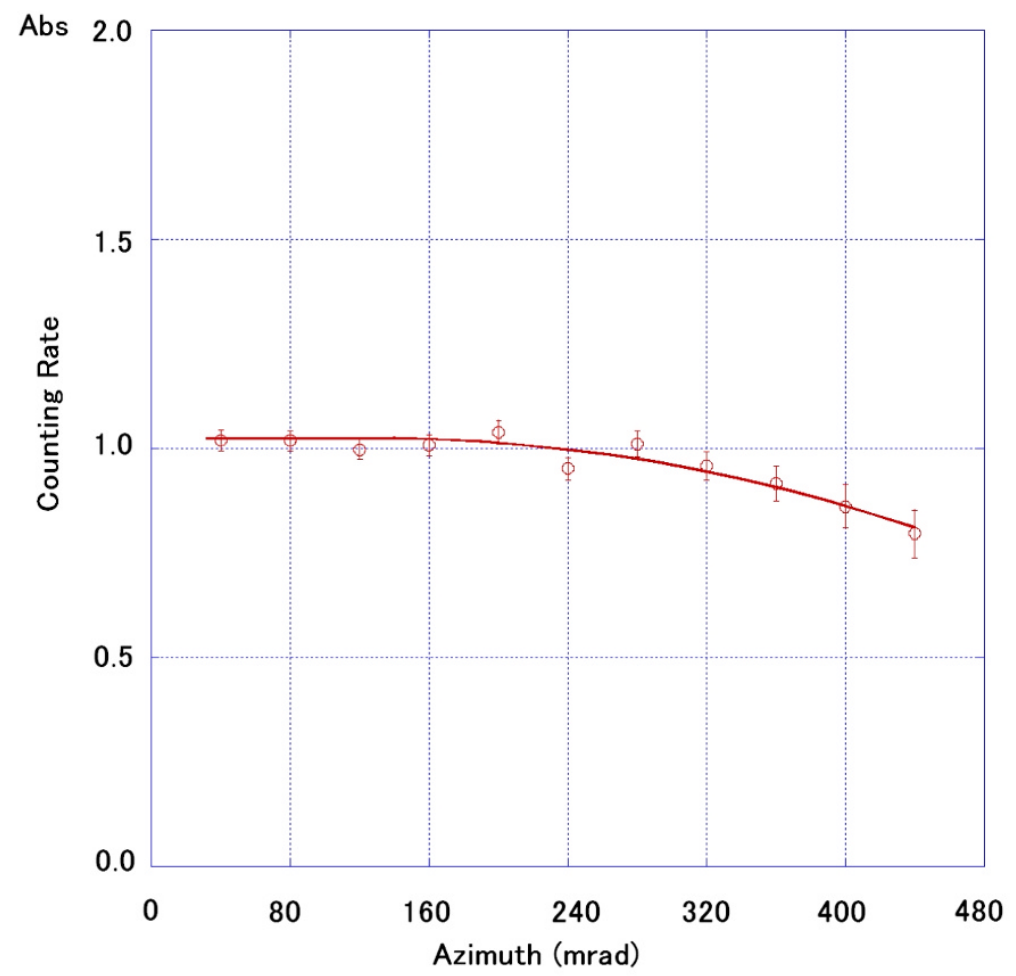

Fig. 3. Count rate of cosmic-ray muons as a function of the distance $(X)$ of the hitting point from the PMT. The ratio of mean pulse height for a minimum ionizing particle and the threshold is $\sim 10$ and $\sim 30$, respectively. Count rate of cosmic-ray muons is normalized to that measured at $X=0$.

of the 1-m long scintillator and then measured the transmission of photons through the scintillator by counting the number of coincidence events between two detector planes for various azimuth angles. The cut ends of the scintillator were coupled with PMTs using a standard optical cou- pling procedure. As shown in Fig. 3(a), the telescope has an angular-dependent detection efficiency that arises from the difference in detection areas and solid angles (geometrical detection efficiency) and the light attenuation in the scintillator for different muon arriving angles. Because muons 
distribute azimuthally isotropic, after correcting the geometrical detection efficiency, we can measure the detection efficiency due to the light attenuation as a function of azimuth angles. The result is shown in Fig. 3 as a function of azimuth angles. Because the ratio of mean pulse height and the threshold is approximately 30 and the attenuation length is measured to be shorter than $1 \mathrm{~m}$, only a slight decrease in the number of events can be seen for the combination that includes the opposite end of the scintillator.

\section{Future Prospects}

Our testing of this assembly demonstrates the following important features regarding the cosmic-ray muon radiography of a volcano: (1) for a cosmic-ray muon detector with dimensions of less than $1 \times 1 \mathrm{~m}$, the detector can be used without any correction for the light attenuation length of the current scintillator; (2) for a detector with dimensions larger than $1 \times 1 \mathrm{~m}$, it is necessary to correct for the light attenuation length of the present scintillator, and the number of events are reduced. A longer attenuation length is needed in order to construct a telescope with a large detection area. Based on the test measurements reported here, we can estimate a realistic enlargement of the detection area by considering the following factors: (1) doping of a small amount of antioxidants or $\mathrm{N}_{2}$-gas purging; (2) use of a vacuum container when casting a scintillator; (3) improving the annealing temperature and time.

\section{Conclusion}

It was evident during all steps of our test measurements of our new scintillator that this scintillator can be used for cosmic-ray muon radiography of a volcano. The synthesis method described here is sufficiently simple and inexpensive to be expanded into a much larger scale. This would contribute to time-dependent measurements as well as an extension to three-dimensional tomography.
Acknowledgments. Special funding arrangements by S. Okubo, K. Nakatsuka, and related people of ERI, JSPS (Japanese Society of Promotion of Science) and JST (Japan Science and Technology Agency) are acknowledged. T. Koyaguchi of ERI, K. Nagamine of UCR, I. Yokoyama, M.J.A., F. Yamashita, and S. Steigerwald are also acknowledged for their valuable suggestions. This work is greatly benefited from useful comments by two reviewers of this manuscript.

\section{References}

Tanaka, H. K. M. and I. Yokoyama, Muon radiography and deformation analysis of the lava dome formed by the 1944 eruption of Usu, Hokkaido-Contact between high-energy physics and volcano physics—, Proc. Jpn. Acad., Ser B, 84, 107-116, 2008.

Tanaka, H. K. M., T. Nakano, S. Takahashi, J. Yoshida, and K. Niwa, Development of an emulsion imaging system for cosmic-ray muon radiography to explore the internal structure of a volcano, Mt. Asama, Nucl. Inst. Meth. Phys. Res. A, 575, 489-497, 2007a.

Tanaka, H. K. M., T. Nakano, S. Takahashi, J. Yoshida, M. Takeo, J. Oikawa, T. Ohminato, Y. Aoki, E. Koyama, H. Tsuji, and K. Niwa, High resolution imaging in the inhomogeneous crust with cosmic-ray muon radiography: The density structure below the volcanic crater floor of Mt. Asama, Japan, Earth Planet. Sci. Lett., 263, 104-113, 2007 b.

Tanaka, H. K. M., T. Nakano, S. Takahashi, J. Yoshida, H. Ohshima, T. Maekawa, H. Watanabe, and K. Niwa, Imaging the conduit size of the dome with cosmic-ray muons: The structure beneath Showa-Shinzan Lava Dome, Japan, Geophys. Res. Lett., 34, L22311, 2007c.

Tanaka, H. K. M., T. Nakano, S. Takahashi, J. Yoshida, M. Takeo, J. Oikawa, T. Ohminato, Y. Aoki, E. Koyama, H. Tsuji, H. Ohshima, T. Maekawa, H. Watanabe, and K. Niwa, Radiographic imaging below a volcanic crater floor with cosmic-ray muons, Am. J. Sci., 308, 843-850, 2008.

Tanaka, H. K. M., T. Uchida, M. Tanaka, H. Shinohara, and H. Taira, Development of a portable assembly-type cosmic-ray muon module for measuring the density structure of a column of magma, Earth Planets Space, 62, this issue, 119-129, 2010.

Y. Yamashina, T. Yamashina, H. Taira, and H. K. M. Tanaka (e-mail: ht@postman.riken.jp) 